\title{
Nuclear imaging in cardiac amyloidosis
}

\author{
A. W. J. M. Glaudemans • R. H. J. A. Slart • \\ C. J. Zeebregts • N. C. Veltman • R. A. Tio • \\ B. P. C. Hazenberg • R. A. J. O. Dierckx
}

Received: 21 August 2008 / Accepted: 26 November 2008 / Published online: 21 January 2009

(C) The Author(s) 2009. This article is published with open access at Springerlink.com

\begin{abstract}
Amyloidosis is a disease characterized by depositions of amyloid in organs and tissues. It can be localized (in just one organ) or systemic. Cardiac amyloidosis is a debilitating disease and can lead to arrhythmias, deterioration of heart function and even sudden death. We reviewed PubMed/Medline, without time constraints, on the different nuclear imaging modalities that are used to visualize myocardial amyloid involvement. Several SPECT tracers have been used for this purpose. The results with these tracers in the evaluation of myocardial amyloidosis and their mechanisms of action are described. Most clinical evidence was found for the use of ${ }^{123}$ I-MIBG. Myocardial defects in MIBG activity seem to correlate well with impaired cardiac sympathetic nerve endings due to amyloid deposits. ${ }^{123}$ I-MIBG is an attractive option for objective evaluation of cardiac sympathetic level and may play an important role in the indirect measurement of the effect of
\end{abstract}

A. W. J. M. Glaudemans $(\bowtie) \cdot$ R. H. J. A. Slart • N. C. Veltman • R. A. J. O. Dierckx

Department of Nuclear Medicine and Molecular Imaging, University Medical Center Groningen,

Hanzeplein 1, P.O. Box 30001, 9700 RB Groningen,

The Netherlands

e-mail: a.w.j.m.glaudemans@ngmb.umcg.nl

C. J. Zeebregts

Department of Surgery (Division of Vascular Surgery),

University Medical Center Groningen,

Groningen, The Netherlands

R. A. Tio

Department of Cardiology, University Medical Center Groningen, Groningen, The Netherlands

B. P. C. Hazenberg

Department of Rheumatology and Clinical Immunology, University Medical Center Groningen,

Groningen, The Netherlands amyloid myocardial infiltration. Other, less sensitive, options are ${ }^{99 \mathrm{~m}} \mathrm{Tc}$-aprotinin for imaging amyloid deposits and perhaps ${ }^{99 \mathrm{~m}} \mathrm{Tc}$-labelled phosphate derivatives, especially in the differential diagnosis of the aetiology of cardiac amyloidosis. PET tracers, despite the advantage of absolute quantification and higher resolution, are not yet well evaluated for the study of cardiac amyloidosis. Because of these advantages, there is still the need for further research in this field.

Keywords Amyloidosis · Nuclear imaging · Cardiology MIBG $\cdot$ Cardiology $\cdot$ Molecular imaging

\section{Introduction}

Amyloidosis is a disease characterized by extracellular tissue deposition of fibrils composed of low molecular weight subunits of a variety of serum proteins (amyloid). Deposition of amyloid can be localized (restricted to one organ or site of the body) or systemic (in various organs and tissues throughout the body). Clinical manifestations are based on the site of the amyloid deposits and are related to the type of precursor protein involved. Systemic amyloidosis is usually fatal and is the cause of about 1 per 1,000 deaths in developed countries. About 3,000 new cases are diagnosed annually in the United States, but amyloidosis is probably underdiagnosed in the elderly population.

Cardiac amyloidosis is described as involvement of the heart by amyloid deposition, whether as part of systemic amyloidosis (as is most commonly the case) or as a localized phenomenon [1]. This can lead to severe arrhythmias, a quick deterioration of heart function, or even sudden death. Several imaging modalities have been 
used to diagnose and identify the extent of amyloid deposition in the heart. No single modality has evolved into a "gold standard". Diagnostic imaging of the severity of the cardiac amyloidosis is important and valuable in the decision making concerning implantable cardioverter defibrillator (ICD) implants or heart transplantation and risk criteria prior to liver transplantation (LT), especially in the hereditary form of amyloidosis (ATTR) caused by a mutation of the precursor protein transthyretin (TTR). Therefore, reliable early detection and measurement of cardiac involvement are invaluable.

We performed a bibliometric search on MEDLINE (PubMed) to evaluate the different imaging modalities used to visualize myocardial amyloid involvement (see Methods). In a recent review, Selvanayagam et al. described nonnuclear imaging modalities. Echocardiography may show several features that are suggestive of cardiac amyloidosis such as thickening of the left ventricular (LV) wall, increased echogenicity of the myocardium, reduced systolic function, thickened valves and a small pericardial effusion. However, the classical features are commonly present only in the later stages of disease. Typical findings on the electrocardiogram are low voltages and a pseudoinfarct pattern, neither of which are very specific for cardiac amyloidosis. Cardiovascular magnetic resonance imaging may offer promise for the early detection of cardiac involvement using the late gadolinium enhancement technique, but this has yet to be proved [1].

Specifically we concentrated on the nuclear imaging modalities, because of their ability to identify pathophysiological activity. This makes it possible to identify organs at risk at an early stage before structural changes have occurred. Nuclear imaging modalities have also the value of reproducible quantification and can even monitor whether therapy is successful. In addition to reviewing the literature, we also wanted to identify the optimal imaging regime to identify amyloidosis patients at risk of cardiac involvement.

\section{Amyloid subtypes}

There are several subtypes of amyloidosis based on the type of protein involved and/or the aetiology for the formation of protein fibrils. Four major systemic types of amyloidosis have been identified [2]:

1. AA type amyloidosis may complicate chronic inflammatory diseases. Serum amyloid A protein, an acute phase reactant, is the precursor protein. Most frequently observed (about 90\%) are renal manifestations (proteinuria and renal failure. Less frequently seen are autonomic neuropathy, hepatomegaly and cardiomyopathy.

2. AL type amyloidosis develops due to deposition of protein derived from immunoglobulin light chain frag- ments produced by a plasma cell dyscrasia. Clinical manifestations are very diverse and depend on the severity of deposition in the various organs and tissues.

3. ATTR type amyloidosis may be hereditary or agerelated (senile). The hereditary form is caused by an autosomal-dominant mutation of the precursor protein TTR. More than 80 mutations have been described. Prominent clinical manifestations are peripheral and autonomic neuropathy, but cardiomyopathy and renal failure have also been observed. Severe cardiomyopathy may be the presenting sign in some of the TTR mutations. In the very old, normal TTR can also behave as precursor protein ('wild-type'). This occurs in $25 \%$ of patients over the age of 80 years and is characterized by a slowly progressive cardiomyopathy.

4. A $32 M$ type amyloidosis. Patients with chronic renal failure and haemodialysis for a long time may suffer from a decreased clearance and deposition of $\beta 2$-microglobulin in bones and joints.

Beside these four major groups several other types have been discovered [3] (see Table 1).

\section{Cardiac amyloidosis}

Cardiac morbidity is based on several pathophysiological mechanisms which occur as a consequence of myocardial deposits of amyloid. Large amounts of deposits may lead to myocyte dysfunction, which is a result of a mechanical obstruction. This may lead to systolic or diastolic dysfunction and symptoms of heart failure. Deposits in the conduction system can lead to dysrhythmias as a result of neuronal dysfunction and to syncope due to arrhythmia or even heart block.

Infiltration of amyloid in the atria as well as the ventricles may cause atrial dysfunction and electromechanical dysfunction of the atrial electrical activity (e.g. atrial fibrillation). This may predispose to thromboembolism. Accumulation of amyloid in the coronary arteries may explain the manifestation of angina or infarction. Cardiac involvement is predominantly present in AL type amyloidosis. Up to $50 \%$ of patients suffer from cardiac manifestations and $25 \%$ show congestive heart failure [4]. Atrial fibrillation and conduction abnormalities are also common. Cardiac involvement is less common in AA type amyloidosis. Less than $5 \%$ of patients with this type have cardiac involvement [5]. In ATTR type amyloidosis cardiac involvement is as frequent as in AL type but leads more rarely to LV systolic dysfunction and clinical congestive heart failure. Problems that occur are cardiac denervation, diastolic dysfunction and atrioventricular block. The problems associated with ATTR types vary with the specific mutation variant [6]. Some mutations are invariably 
Table 1 Amyloid types

\begin{tabular}{|c|c|c|c|}
\hline Amyloid type & Protein precursor & Systemic (S)/Localized (L) & Clinical features \\
\hline AA & Serum amyloid A & $\mathrm{S}$ & $\begin{array}{l}\text { Reactive; renal failure, proteinuria, } \\
\text { hepatomegaly, neuropathy, cardiomyopathy }(<5 \%)\end{array}$ \\
\hline $\mathrm{AL}$ & $\begin{array}{l}\text { Immunoglobulin } \\
\text { light chain }\end{array}$ & $\mathrm{S}, \mathrm{L}$ & $\begin{array}{l}\text { Plasma cell dyscrasia; all kinds of manifestations, } \\
\text { cardiomyopathy }(26-50 \%)\end{array}$ \\
\hline ATTR & Transthyretin & $\mathrm{S}$ & $\begin{array}{l}\text { Hereditary or age-related (senile); neuropathy, } \\
\text { cardiomyopathy, renal failure }\end{array}$ \\
\hline $\mathrm{A} \beta 2 \mathrm{M}$ & $\beta 2$-Microglobulin & $\mathrm{S}$ & After chronic dialysis; bone and joint problems \\
\hline $\mathrm{AH}$ & $\begin{array}{l}\text { Immunoglobulin } \\
\text { heavy chain }\end{array}$ & $\mathrm{S}, \mathrm{L}$ & Plasma cell dyscrasia; all kinds of manifestations \\
\hline AApoAI & Apolipoprotein AI & $\mathrm{S}, \mathrm{L}$ & Hereditary; neuropathy, nephropathy, aorta (local) \\
\hline AApoAII & Apolipoprotein AII & $\mathrm{S}$ & Hereditary \\
\hline AApoAIV & Apolipoprotein AIV & $\mathrm{S}$ & Sporadic, associated with ageing \\
\hline AGel & Gelsolin & $\mathrm{S}$ & Hereditary \\
\hline ACys & Cystatin $\mathrm{C}$ & $\mathrm{S}$ & Hereditary; cerebral haemorrhage \\
\hline ALys & Lysozyme & $\mathrm{S}$ & Hereditary \\
\hline AFib & Fibrinogen & $\mathrm{S}$ & Hereditary, renal failure \\
\hline ABri & ABripP & $\mathrm{S}$ & Familial dementia, British \\
\hline ADan & ADanPP & $\mathrm{L}$ & Familial dementia, Danish \\
\hline $\mathrm{A} \beta$ & $\mathrm{A} \beta$ protein precursor & $\mathrm{L}$ & Alzheimer's disease, ageing \\
\hline ACal & (Pro)calcitonin & $\mathrm{L}$ & Medullary carcinomas of the thyroid \\
\hline AANF & Atrial natriuretic factor & $\mathrm{L}$ & Isolated atrial amyloid \\
\hline AIAPP & Islet amyloid polypeptide & $\mathrm{L}$ & Islet of Langerhans, diabetes type II, insulinoma \\
\hline APrP & Prion protein & $\mathrm{L}$ & Spongiform encephalopathies \\
\hline APro & Prolactin & $\mathrm{L}$ & Ageing pituitary, prolactinomas \\
\hline AIns & Insulin & $\mathrm{L}$ & Iatrogenic \\
\hline AMed & Lactadherin & $\mathrm{L}$ & Senile aortic, media \\
\hline AKer & Kerato-epithelin & $\mathrm{L}$ & Cornea, familial \\
\hline ALac & Lactoferrin & $\mathrm{L}$ & Cornea \\
\hline
\end{tabular}

associated with cardiac disease, others usually only with neurological disease, and many a combination of both. The senile form often has cardiac involvement and is related with congestive heart failure and conduction abnormalities.

Cardiac involvement in AL type amyloidosis often leads to significant rapidly progressive cardiac dysfunction, while the ATTR types are typically associated with milder clinical manifestations and slower progression [7].

\section{Methods}

For collecting imaging studies we used MEDLINE (PubMed) to search the literature using the following search terms 'myocard*' AND 'amyloid*' AND 'imaging'. This produced 114 results. These results were manually scanned and articles were excluded based on the following criteria: amyloid was not the main topic, but only an example; 'amyloid' and 'myocard' were unrelated in the article; language other than English, German or Dutch. This resulted in 55 studies of a nuclear imaging modality.

In addition, for every imaging modality a search was performed with the terms 'myocard*' AND 'amyloid*'
AND 'name of the imaging modality'. This resulted in a further seven articles. In total, we found 62 studies (59 SPECT, 3 PET). The SPECT studies were classified based on the physiological mechanism that was visualized (see Table 2). Finally, each imaging modality was reviewed in terms of its results.

Table 2 Nuclear imaging modalities for cardiac amyloidosis

\begin{tabular}{ll}
\hline Physiological mechanism & Nuclear imaging (SPECT) modality \\
\hline Innervation & ${ }^{123} \mathrm{I}$-MIBG (7) \\
Metabolism & ${ }^{123} \mathrm{I}$-BMIPP (2) \\
& ${ }^{99 \mathrm{~m}} \mathrm{Tc}(\mathrm{V})-\mathrm{DMSA}(3)$ \\
Perfusion & ${ }^{99 \mathrm{~m}} \mathrm{Tc}-$ sestamibi (2) and ${ }^{201} \mathrm{Tl}(6)$ \\
Amyloid-deposits & ${ }^{99 \mathrm{~m}} \mathrm{Tc}-$ aprotinin (3) \\
& ${ }^{99 \mathrm{~m}} \mathrm{Tc}-($ pyro)phosphate (27) \\
& ${ }^{67} \mathrm{Ga}(4)$ \\
Ventricular function & ${ }^{111} \mathrm{In}-$ antimyosin antibody (2) \\
\hline
\end{tabular}




\section{Nuclear imaging modalities}

Innervation

\section{${ }^{123} I-M I B G$}

At present, the most commonly used SPECT tracer to assess cardiac neurotransmission is ${ }^{123} \mathrm{I}-\mathrm{MIBG}$ [8]. It is widely accepted as a good method for assessing myocardial sympathetic nerve activity and innervation in a variety of cardiac disease states and the effect of treatment with cardiovascular drugs in chronic heart failure [9].

MIBG (metaiodobenzylguanidine) is an analogue of the "false" neurotransmitter guanetidine, a potent neuronblocking agent that acts selectively on sympathetic nerves. MIBG has a molecular structure similar to that of noradrenaline and shares the same uptake and storage mechanisms in the sympathetic nerve endings [8].

On the other hand, in contrast to noradrenaline, MIBG undergoes almost no metabolism via enzymes. So MIBG is retained in the sympathetic nerve endings and the localiza- tion of MIBG relates to the presence of sympathetic nerves. When labelled with ${ }^{123} \mathrm{I}$, it can be used to image the sympathetic nervous system in various organs, and has an important role in myocardial imaging [10].

The most common indices are the heart to mediastinum $(\mathrm{H} / \mathrm{M})$ ratio and the washout rate obtained from anterior planar images. Early images are obtained 15 to $30 \mathrm{~min}$ after injection, delayed images $4 \mathrm{~h}$ later (see Fig. 1). The accumulation on the early MIBG image reflects the distribution of cardiac sympathetic nerves and the uptake function at the nerve endings, while the washout rate is an index of the degree of sympatheticotonia. The rate of uptake of MIBG in the myocardium is as low as $1-2 \%$. It is possible to obtain a mean value for the whole $\mathrm{LV}$, but also for the various regions by analysis on a polar map.

The use of MIBG in patients with amyloidosis was reported for the first time by Nakata et al. in 1995, who found decreased myocardial activity of ${ }^{123} \mathrm{I}-\mathrm{MIBG}$ in all cardiac regions in a patient with severe peripheral neuropathy due to a TTR-related familial amyloidotic polyneuropathy (FAP). Nakata et al. were the first to suggest the

Fig. 1 MIBG uptake in the heart after $15 \mathrm{~min}($ left $)$ and after $4 \mathrm{~h}$ (right). The H/M ratio was 3.06 after $15 \mathrm{~min}$ and 2.65 after $4 \mathrm{~h}$. The washout was $13 \%$

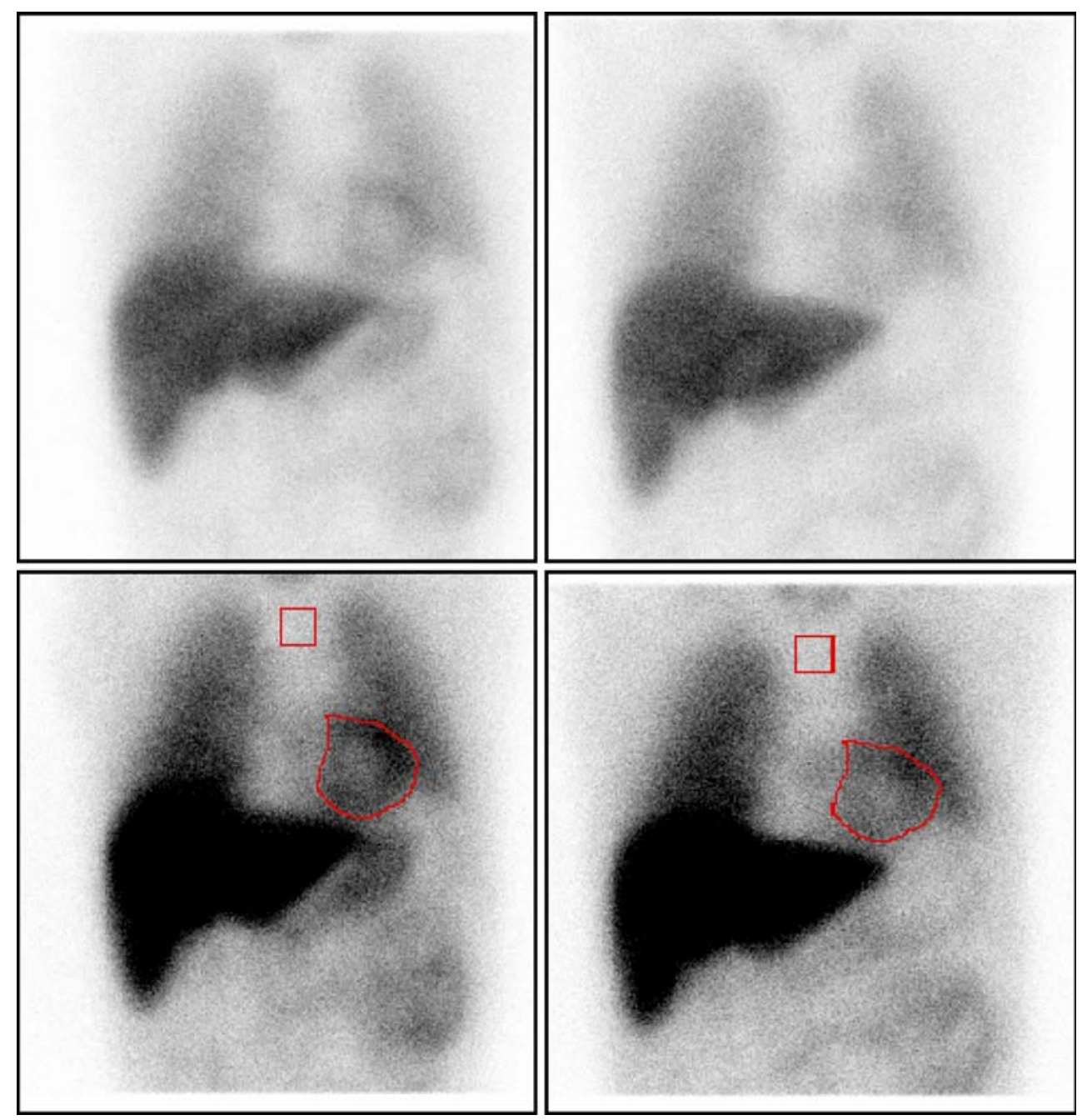


hypothesis that a myocardial defect of MIBG activity provides direct evidence of impaired cardiac sympathetic nerve endings due to amyloid deposits in FAP [11]. This finding was confirmed by Arbab et al. in 1997, who also reported almost no uptake of ${ }^{123}$ I-MIBG in the heart of a man with FAP. This patient underwent several investigations. ${ }^{99 \mathrm{~m}} \mathrm{Tc}(\mathrm{V})$-DMSA showed some myocardial involvement of amyloidosis, and ${ }^{201} \mathrm{Tl}$ and ${ }^{123} \mathrm{I}$-BMIPP showed normal blood perfusion and normal fatty acid metabolism, respectively (all these tracers are discussed below in this review). The decreased uptake of MIBG in the heart indicated a severe impairment of sympathetic function [12].

In 1997, Tanaka et al. were the first to perform a prospective study to assess myocardial sympathetic innervation using ${ }^{123}$ I-MIBG imaging in patients with FAP and compared it with other imaging modalities. They performed echocardiography, ${ }^{123} \mathrm{I}-\mathrm{MIBG},{ }^{201} \mathrm{Tl}$ and ${ }^{99 \mathrm{~m}} \mathrm{Tc}$-PYP (discussed below) imaging of the heart in 12 patients with FAP and biopsy-proven cardiac amyloidosis. LV wall thickening on echocardiography was observed in 4 of 10 patients who had no clinical evidence of overt heart disease. Eight of the 12 patients showed no myocardial MIBG accumulation, with limited uptake in the remaining 4 only in the LV anterior wall. Diffuse but mild uptake of $99 \mathrm{~m}$ Tc-PYP occurred in only 4 of the 12 patients, and all 12 had normal results on ${ }^{201} \mathrm{Tl}$ scanning. They concluded that patients with FAP show a high incidence of myocardial denervation with viable myocardium that can be identified in early stage cardiac amyloidosis [13].

Another clinical trial was performed by Delahaye et al. who studied 17 patients with biopsy-proven FAP by ${ }^{123} \mathrm{I}$ MIBG, heart rate variability analysis, coronary angiography, radionuclide ventriculography, rest ${ }^{201} \mathrm{Tl}$ SPECT and echocardiography. Coronary arteries, LV systolic function and rest ${ }^{201} \mathrm{Tl}$ studies were normal in all patients. On echocardiographic examination evidence of only mild amyloid infiltration was found. Cardiac MIBG uptake was dramatically decreased in patients compared with agematched control subjects (H/M ratio at $4 \mathrm{~h}: 1.36 \pm 0.26$ vs. $1.98 \pm 0.35, p<0.001)$. There was no difference in washout rate. The clinical severity of the polyneuropathy correlated with the decrease in MIBG uptake at $4 \mathrm{~h}$. So patients with FAP have sympathetic cardiac denervation as assessed by MIBG imaging despite a preserved LV systolic function and cardiac perfusion [14]. These findings are in agreement with the results of Tanaka et al.

In $\mathrm{AL}$ (primary) amyloidosis, intractable congestive heart failure is the most common symptom (occurring in $26-30 \%$ of patients), in which interstitial amyloid deposits are severe enough to cause fatal cardiac dysfunction [15]. In 2002, Hongo et al. were the first to test the hypothesis that myocardial sympathetic nerve innervation might be impaired and relates to the presence or absence of clinical autonomic abnormalities and congestive heart failure in $\mathrm{AL}$ amyloidosis. They examined 25 patients using ${ }^{123} \mathrm{I}-\mathrm{MIBG}$ scintigraphy. The diagnosis of AL amyloidosis was made on the basis of histological evidence of systemic amyloidosis and an association with plasma cell dyscrasia and/or identification of an immunoglobulin light chain in the serum or urine. Of the 25 patients, 16 were without autonomic symptoms and 9 had autonomic neuropathy. The H/M ratio at $3 \mathrm{~h}(1.53 \pm 0.06$ vs. $1.29 \pm 0.05, p<0.001)$ and the myocardial washout ratio $(41.5 \pm 4.8 \%$ vs. $30.8 \pm 4.0 \%$, $p<0.001$ ) of MIBG were significantly higher in patients without autonomic symptoms than in those showing autonomic neuropathy. Patients demonstrating congestive heart failure (10 of 16 patients without autonomic symptoms and 5 of 9 patients with autonomic neuropathy) showed a significantly decreased $\mathrm{H} / \mathrm{M}$ ratio and an increased washout compared to patients without heart failure. So, in conclusion, patients with $\mathrm{AL}$ amyloidosis and no autonomic dysfunction showed variable degrees of enhanced cardiac neuronal activity with presynaptic sympathetic dysfunction. In contrast, patients with AL amyloidosis and autonomic neuropathy exhibited prominent myocardial denervation with normal or impaired sympathetic neural function of the heart. This study showed a heterogeneous myocardial uptake and turnover of MIBG in patients with AL amyloidosis and the uptake reflects the presence or absence of congestive heart failure and cardiac autonomic dysfunction [9].

Another study with the use of ${ }^{123}$ I-MIBG was performed by Delahaye et al. in 2006 . They conducted a study to define the outcome of cardiac amyloid involvement after LT in 31 patients with FAP. Patients were evaluated before and after LT ( $24 \pm 15$ months). Diffuse sympathetic denervation was found on MIBG planar imaging in patients evaluated before LT and compared with 12 control subjects (H/M ratio: $1.45 \pm 0.29$ vs. $1.98 \pm 0.35, p<0.001)$. After LT no change in sympathetic innervation was found $(\mathrm{H} / \mathrm{M}$ ratio after LT: $1.46 \pm 0.28$ ), whereas other symptoms (conduction disturbances, ventricular arrhythmias and LV wall thickening) showed progression of cardiac amyloid infiltration. The severity of cardiac sympathetic denervation found in FAP patients before LT was associated with a neurological worsening after LT. Therefore, an objective measurement of cardiac adrenergic innervation, such as that obtained by MIBG imaging, could help guide the indications for LT in FAP patients in the early stages of the disease [16].

In most studies, the explanation of the decreased MIBG uptake was denervation because of the presence of amyloid deposits. Another cause of the decrease in MIBG uptake may be a specific involvement of the autonomic system, as found by Delahaye et al. in 2001. They used the PET tracer ${ }^{11} \mathrm{C}$-methylquinuclidinyl benzilate (MQNB) and found a parasympathetic myocardial denervation with a very low increase in heart rate in patients after atropine injection 
compared with controls, and an upregulation of myocardial parasympathetic postsynaptic muscarinic receptors in response to the presynaptic denervation [17].

Overall, ${ }^{123}$ I-MIBG seems a good option for objective evaluation of cardiac sympathetic level and it also seems to have a role in the indirect measurement of amyloid myocardial infiltration.

\section{Metabolism}

\section{${ }^{123} I-B M I P P$}

As free fatty acids are the main energy source of the heart under aerobic fasting conditions, evaluation of myocardial fatty acid metabolism is useful in understanding pathophysiological conditions in various heart diseases. The instability of labelling and the rapid catabolism in cardiac myocytes through the $\beta$ oxidative pathway have precluded the use of ${ }^{123}$ I-labelled fatty acids for SPECT. An analogue of free fatty acid, $\beta$-methyl-iodophenyl pentadecanoic acid (BMIPP), is trapped mainly in the triglyceride fractions and is metabolized more slowly by $\beta$ oxidation. Because of its prolonged retention in the myocardium it is suitable for SPECT imaging [18]. This tracer is generally used for the evaluation of ischaemic heart disease $[19,20]$ and cardiomyopathies $[18,21]$. In jeopardized but viable myocardium BMIPP uptake is less than perfusion (mismatch), and in nonviable tissue a matched pattern of decreased uptake of both tracers is visible [22].

For cardiac amyloidosis imaging, the use of ${ }^{123}$ I-BMIPP is limited to only one case report. In 1997 Arbab et al. used various myocardial SPECT agents in a patient with FAP and suspected myocardial involvement. The ${ }^{123}$ I-BMIPP study showed normal fatty acid metabolism, whereas ${ }^{123}$ I-MIBG showed a severe impairment of sympathetic function.

This case report suggests that ${ }^{123}$ I-BMIPP although useful for evaluating the fatty acid metabolism in the heart has no role in the evaluation of cardiac amyloidosis.

\section{${ }^{99 m} T c(V)-D M S A$}

\footnotetext{
${ }^{99 \mathrm{~m}} \mathrm{Tc}$ pentavalent dimercaptosuccinic acid (V-DMSA) is a well-known tumour-seeking agent that accumulates in medullary thyroid cancer and soft-tissue tumours [23]. Its mechanism of accumulation is thought to be related to the structural similarity between pentavalent DMSA and the phosphate ion $\mathrm{PO}_{4}{ }^{3-}$, which is avidly taken up by some cancer cells [24]. Its usefulness in the evaluation of amyloidosis associated with plasmacytoma (AL type) has been reported [25, 26]. A disadvantage of pentavalent DMSA is its physiological uptake in the blood pool, which explains the very limited use of pentavalent DMSA for the imaging of amyloid deposits in the heart.
}

A patient with histologically proven dilated cardiomyopathy and a patient with clinically diagnosed cardiomyopathy (cardiac amyloidosis was strongly suspected but was not confirmed) were examined with ${ }^{99 \mathrm{~m}} \mathrm{Tc}(\mathrm{V})$-DMSA by Ohta et al. ${ }^{99 \mathrm{~m}} \mathrm{Tc}(\mathrm{V})$-DMSA accumulation was clearly demonstrated in the damaged myocardium. These results suggested that ${ }^{99 \mathrm{~m}} \mathrm{Tc}(\mathrm{V})$-DMSA could be used as a useful agent for the evaluation of cardiomyopathy [26].

Another case report was presented by Arbab et al. who used various SPECT agents in a patient with FAP and suspected myocardial involvement. They acquired 24-h SPECT images to avoid the blood pool activity in the heart. The SPECT images showed abnormal uptake of the tracer in the heart. They suggested that in this patient either ${ }^{99 \mathrm{~m}} \mathrm{Tc}$-DMSA or ${ }^{123} \mathrm{I}$-MIBG may be useful to detect involvement of the heart by the amyloid process [12].

In conclusion, these case reports suggest a role for pentavalent DMSA in the imaging of cardiac amyloidosis. However, the physiological uptake in the blood pool is a disadvantage.

\section{Perfusion}

\section{${ }^{99 m} T c$-sestamibi and ${ }^{201} \mathrm{Tl}$}

Both sestamibi and ${ }^{201} \mathrm{Tl}$ are well-known perfusion tracers. They perform well in clinical terms, with high sensitivity and specificity for detecting ischaemia. The use of both tracers for cardiac amyloid deposition is limited to a couple of case reports [27, 28] and one rest-redistribution study with ${ }^{201} \mathrm{Tl}$.

Wechalekar et al. found uptake of a bone-seeking agent in the heart, suggesting an infiltrative heart disease. ${ }^{99 \mathrm{~m}} \mathrm{Tc}$ Sestamibi myocardial perfusion scintigraphy showed no evidence of ischaemia but a low ejection fraction of $28 \%$, also suggesting the possibility of a muscle disorder. Later, the patient was diagnosed with cardiac amyloidosis [29]. Kobayashi et al. assessed the changes in cardiac condition in a patient with biopsy-proven cardiac amyloidosis by means of SPECT imaging of the heart perfusion with ${ }^{201} \mathrm{Tl}$ and imaging with a bone-seeking agent on admission and 3 months later. The ${ }^{201} \mathrm{Tl}$ scintigraphy remained almost unchanged with defects in the septal and posterior wall regions, the bone-seeking agent showed a marked increase in myocardial accumulation along with deterioration of symptoms and signs. The authors considered that ${ }^{201} \mathrm{Tl}$ defects might reflect myocyte damage due to amyloid deposits in the myocardium and that the combination of both a perfusion and a bone-seeking agent is of value in assessing the degree and distribution of the infiltrative process of cardiac amyloidosis [30].

Kodama et al. performed rest-redistribution ${ }^{201} \mathrm{Tl}$ myocardial scintigraphy in five patients with biopsy-proven systemic amyloidosis with cardiac involvement and in 12 
controls. Histopathological study in amyloid heart demonstrated that myocytes destroyed by deposition of amyloid protein are present together with viable myocytes. Rapid $\mathrm{Tl}$ washout may be found in amyloid heart as well as in regions which have a mixture of viable myocytes and scar tissue in patients with myocardial infarction. They found that the mean washout rate of the whole heart was higher in the amyloidosis patients than in the control group. Four of the five patients in the amyloidosis group showed a very high washout rate. They also died within a year. They suggested that the washout rate may reflect the severity of amyloid deposits in the myocardium and may provide prognostic information [31].

Altogether, the role for perfusion tracers in evaluating cardiac amyloidosis is limited.

Amyloid deposits

${ }^{99 m}$ Tc-aprotinin

Antiproteases have been detected in amyloid deposits [31]. Although the significance of the accumulation of these Antiproteases has not been clarified, this observation led to the investigation of the binding of a protease inhibitor labelled with ${ }^{99 \mathrm{~m}} \mathrm{Tc}$ to amyloid deposits [32]. Aprotinin, a low molecular weight polypeptide protease inhibitor, was found to fulfil this criterion. Because of its physiological uptake in the liver, spleen and kidneys it is only useful in extraabdominal disease [32].

In 1995, Aprile et al. were the first to evaluate ${ }^{99 \mathrm{~m}} \mathrm{Tc}$ aprotinin for the imaging of amyloid deposits. They reviewed the scintigraphic findings in 24 patients with $\mathrm{AL}$ amyloidosis and 1 patient with a hereditary form who were known to have extraabdominal involvement. Myocardial accumulation was found in 11 patients. Unfortunately, no endomyocardial biopsy, the gold standard, was performed to confirm this finding. The authors concluded that ${ }^{99 \mathrm{~m}} \mathrm{Tc}-$ aprotinin is a potential tracer for the detection of extraabdominal AL amyloid deposits, including heart deposits, which are of paramount prognostic relevance, and that further investigations are mandatory [33].

Despite these promising results, it was 7 years before publication of the next article in 2002 by Schaadt et al. They found focal accumulations of ${ }^{99 \mathrm{~m}} \mathrm{Tc}$-aprotinin in different organs of 22 patients with a total of 90 lesions, of which 20 were confirmed by biopsy or autopsy. Accumulation in the myocardium was seen in 8 patients, confirmed by biopsy in $4 .{ }^{99 \mathrm{~m}} \mathrm{Tc}$-aprotinin was found to be a fairly sensitive and specific diagnostic modality in patients with suspected amyloidosis and is useful for detection of a wide range of lesions [34].

A recent study was reported by Han et al. in 2007. They retrospectively reviewed 35 patients who underwent ${ }^{99 \mathrm{~m}} \mathrm{Tc}$ - aprotinin scans for amyloid imaging, of whom 18 had biopsy-proven amyloidosis (14 AL, 3 AA and 1 ATTR). The main sites were renal (eight), cardiac (five), lung (one), oral-nasal (two) and subcutaneous tissue (two). The remaining 17 patients showed a negative biopsy (eight) or negative clinical and noninvasive tests (nine) and were included as a control group. ${ }^{99 \mathrm{~m}} \mathrm{Tc}$-aprotinin uptake in the region of the myocardium was noted in 5 of the 35 patients with mild or moderate intensity compared with renal activity. These five patients had a final diagnosis of cardiac amyloidosis based on the presence of clinical, echocardiographic and MRI features of infiltrative myocardial disease and the exclusion of other causes of cardiomyopathy. Four of these five patients died from cardiac complications within 2 years of diagnosis. The other 30 patients had no clinical or echocardiographic evidence of cardiac amyloidosis and none showed significant uptake in the heart region. The authors concluded that ${ }^{99 \mathrm{~m}} \mathrm{Tc}$-aprotinin may help in the diagnosis of amyloid disease in the heart [35].

In conclusion, these studies suggest a role for ${ }^{99 \mathrm{~m}} \mathrm{Tc}$-aprotinin in the imaging of cardiac involvement of amyloidosis.

\section{${ }^{99 m}$ Tc-labelled phosphate derivatives}

Back in 1977 the first study was published that associated ${ }^{99 \mathrm{~m}}$ Tc-diphosphonate with amyloid. This association is possibly due to high calcium compounds in amyloid, which suggests the possibility of visualizing calcifications in the amyloid deposits [36]. Since then, several variants of phosphate-derivatives have been labelled with ${ }^{99 \mathrm{~m}} \mathrm{Tc}$ and tested, including ${ }^{99 \mathrm{~m}} \mathrm{Tc}$-pyrophosphate $\left({ }^{99 \mathrm{~m}} \mathrm{Tc}-\mathrm{PYP}\right),{ }^{99 \mathrm{~m}} \mathrm{Tc}$ methylene diphosphonate (MDP) and ${ }^{99 \mathrm{~m}} \mathrm{Tc}-3,3$-diphosphono-1,2-propanodicarboxylic acid (DPD) [37].

Many case reports were published in the early 1980s and later [30, 38-51]. In 1982, Wizenberg et al. investigated a group of ten patients with tissue-proven amyloidosis using ${ }^{99 \mathrm{~m}}$ Tc-PYP (only two had histologically proven cardiac amyloidosis). All patients had intense diffuse cardiac uptake on radionuclide imaging. Other noninvasive tests suggested cardiomyopathic changes. The authors concluded that, when combined with noninvasive tests of LV performance, positive myocardial PYP scanning may provide a new and useful diagnostic method for the evaluation of amyloid heart disease [52].

Falk et al. prospectively performed ${ }^{99 \mathrm{~m}} \mathrm{Tc}-\mathrm{PYP}$ scintigraphy in 20 patients with biopsy-proven primary (AL) amyloidosis. Of these 20 patients, 11 had definitive cardiac involvement compatible with amyloid cardiomyopathy, 9 of whom had symptoms of congestive heart failure. In 9 of the 11 patients a diffuse myocardial pyrophosphate uptake was seen, that did not depend on the presence of congestive heart failure. But of the 9 remaining patients with biopsyproven AL amyloidosis but with a normal echocardiogram, 
only 2 showed uptake in the heart. None of 10 control patients had a positive myocardial uptake [53]. So ${ }^{99 \mathrm{~m}} \mathrm{TC}$ PYP myocardial scanning seems to be a sensitive test for the diagnosis of cardiac amyloidosis in patients with congestive heart failure. However, it does not appear to be of value for the early detection of cardiac involvement in patients with known primary amyloidosis. So the clinical relevance remains uncertain.

In 1983, Lee et al. reported a prospective, comparative study of the efficacy of ${ }^{99 \mathrm{~m}} \mathrm{Tc}-\mathrm{PYP}$ and ${ }^{99 \mathrm{~m}} \mathrm{Tc}-\mathrm{MDP}$ in detecting soft-tissue amyloidosis. The scans were obtained at 10-day intervals in seven patients with histologically proven amyloidosis. All seven patients had abnormal cardiac and/or hepatic ${ }^{99 \mathrm{~m}} \mathrm{Tc}-\mathrm{PYP}$ uptake, compared to only four patients who were injected with ${ }^{99 \mathrm{~m}} \mathrm{Tc}-\mathrm{MDP}$. ${ }^{99 \mathrm{~m}}$ Tc-PYP was found to be a better scanning agent for softtissue amyloidosis and for cardiac localization of amyloid [54]. A year later, Eriksson et al. reported less convincing results for ${ }^{99 \mathrm{~m}} \mathrm{Tc}-\mathrm{PYP}$. They examined 12 patients with familial amyloidosis with polyneuropathy both by crosssectional echocardiography and by ${ }^{99 \mathrm{~m}} \mathrm{Tc}-\mathrm{PYP}$ scintigraphy to assess involvement of the heart noninvasively. All 12 patients had echocardiographic abnormalities, whereas only four patients had abnormal myocardial uptake of ${ }^{99 \mathrm{~m}} \mathrm{Tc}-$ PYP. They concluded that a threshold amount of amyloid is probably required to produce an abnormal scintigram, although lesions with less amyloid can evidently be identified by echocardiography [55].

The findings of Lee et al. were also countered by those of Janssen et al. in 1989. They described the results of scintigraphy with ${ }^{99 \mathrm{~m}} \mathrm{Tc}-\mathrm{MDP}$ and ${ }^{99 \mathrm{~m}} \mathrm{Tc}-\mathrm{PYP}$ as a noninvasive test for the distribution of organ involvement in five different types of amyloidosis. For whole-body imaging, ${ }^{99 \mathrm{~m}} \mathrm{Tc}-\mathrm{MDP}$ seemed to be a sensitive noninvasive screening test to visualize the extent and distribution of organ involvement in systemic AA and AL amyloidosis. Echocardiography, however, was more sensitive for demonstrating cardiac involvement than both phosphate-derivatives. In general, ${ }^{99 \mathrm{~m}} \mathrm{Tc}-\mathrm{MDP}$ images showed a better contrast than ${ }^{99 \mathrm{~m}}$ Tc-PYP images [56].

Hartmann et al. used a combined first-pass and static scintigraphy with ${ }^{99 \mathrm{~m}} \mathrm{Tc}-\mathrm{PYP}$ in seven patients with myocardial amyloidosis. This diagnosis was made if patients had biopsy proven amyloidosis in other tissues, cardiac symptoms and echocardiographic and electrocardiographic features compatible with amyloidosis. The static images showed only insignificant myocardial uptake of the tracer possibly due to less amyloid fibril. The first-pass studies, however, revealed reduced systolic function in four patients and impaired diastolic function in six of the seven patients. They concluded that ${ }^{99 \mathrm{~m}} \mathrm{Tc}$-PYP scintigraphy is not useful in screening patients for cardiac involvement in amyloidosis, but that first-pass studies yield valuable information about diastolic function impairment [57]. Tanaka et al. performed echocardiography and ${ }^{123} \mathrm{I}-\mathrm{MIBG}$, ${ }^{201} \mathrm{Tl}$ and ${ }^{99 \mathrm{~m}} \mathrm{Tc}-\mathrm{PYP}$ imaging of the heart in 12 patients with familial amyloid polyneuropathy and biopsy-proven cardiac amyloidosis. They found diffuse but mild myocardial uptake of ${ }^{99 \mathrm{~m}} \mathrm{Tc}-\mathrm{PYP}$ in only 4 of the 12 patients. ${ }^{123} \mathrm{I}-$ MIBG was found in an earlier stage of cardiac amyloidosis than ${ }^{99 \mathrm{~m}}$ Tc-PYP [13].

More recently (in 2005), Perugini et al. investigated the diagnostic accuracy of ${ }^{99 \mathrm{~m}} \mathrm{Tc}-\mathrm{DPD}$ scintigraphy for the differentiation of AL and ATTR-type cardiac amyloidosis. Myocardial uptake was significantly higher in the ATTR group $(n=15)$ than in the AL group $(n=10)$. Cardiac uptake was visualized in all the ATTR patients but not in any of the AL patients. ${ }^{99 \mathrm{~m}} \mathrm{Tc}-\mathrm{MDP}$ scintigraphy was also performed in 11 patients with myocardial ${ }^{99 \mathrm{~m}} \mathrm{Tc}-\mathrm{DPD}$ uptake. These patients showed no MDP myocardial uptake. The authors concluded that ${ }^{99 \mathrm{~m}} \mathrm{Tc}-\mathrm{DPD}$ scanning is a useful step in the work-up for the differential diagnosis of ATTR versus AL aetiology in patients with documented cardiac amyloidosis [58].

Overall, it seems that imaging with ${ }^{99 \mathrm{~m}} \mathrm{Tc}$-labelled phosphates has no significant role in detecting myocardial amyloidosis. The only exception may be, as suggested by Perugini at al. [58], helping in the differential diagnosis of ATTR versus AL aetiology [58]. Positive phosphate scanning seems to correlate with high amounts of amyloid fibrils in the heart, but this occurs at an advanced stage when clinical symptoms of cardiomyopathy are already apparent. However, be alert when diffuse myocardial uptake is seen on a bone scan - it could be cardiac amyloidosis.

\section{${ }^{67} \mathrm{Ga}$}

In the past, ${ }^{67} \mathrm{Ga}$-citrate was widely used for the detection of various infections and tumours as a nonspecific agent for all kinds of diseases. The mechanisms involved in the concentration of gallium in infections and tumours are still not fully understood, but in almost all cases the concentration of gallium correlates with the expression of the transferrin receptor [59]. With regard to amyloidosis, ${ }^{67} \mathrm{Ga}$ has very limited value.

In 1979, Braun et al. were the first to describe diffuse ${ }^{67} \mathrm{Ga}$ uptake in the myocardium [60]. This finding was confirmed by Montes et al. who found that ${ }^{67} \mathrm{Ga}-$ citrate scintigraphy exhibited marked myocardial accumulation, but not as high as the diffuse and intense uptake of ${ }^{99 \mathrm{~m}} \mathrm{Tc}-\mathrm{PYP}$ in the myocardium [61]. Li et al. also found mild diffuse myocardial uptake of ${ }^{67} \mathrm{Ga}$-citrate in a patient with amyloid cardiomyopathy, but also less intense than the myocardial uptake of ${ }^{99 \mathrm{~m}}$ Tc-PYP [62].

In general, there seems to be no role for ${ }^{67} \mathrm{Ga}$-citrate in detecting cardiac amyloidosis. 


\section{${ }^{111}$ In antimyosin antibody}

${ }^{111}$ In-labelled antimyosin antibodies have been shown to bind specifically to areas of myocardial necrosis. Myosinspecific antibodies bind to damaged myocardial cells [63]. Previous studies have shown antimyosin uptake in patients with myocardial infarction, myocarditis, cardiac transplant rejection and Adriamycin cardiotoxicity [64, 65].

Lekakis et al. reported a case of cardiac amyloidosis detected by antimyosin imaging [66]. They also prospectively investigated the diagnostic value of antimyosin scanning in seven patients with biopsy-proven cardiac amyloidosis. In each patient with cardiac amyloidosis the antimyosin scan was abnormal with a heart/lung $(\mathrm{H} / \mathrm{L})$ ratio ranging from 2.1 to 3.2 , significantly higher than that observed in six control patients (H/L ratio 1.3-1.6). Diffuse uptake of antimyosin was observed in all patients with cardiac amyloidosis. Patients with amyloidosis and congestive heart failure had a higher $\mathrm{H} / \mathrm{L}$ ratio $(2.76 \pm 0.30)$ compared with patients without clinically evident heart failure $(2.17 \pm 0.06)$. On the basis of these observations the authors concluded that LV thickening with diffuse myocardial antimyosin uptake is highly suggestive of amyloid heart disease [67].

Despite these promising results, this is the only article that was found in the literature concerning antimyosin uptake in cardiac amyloidosis.

\section{Ventricular function}

\section{Radionuclide blood pool ventriculography}

Different studies have used radionuclide ventriculography to compare restrictive cardiomyopathy (in some cases due to cardiac amyloid) and pericardial constriction. Aroney et al. found no difference between LV peak filling rates normalized to end-diastolic volume in patients without cardiac disease and in patients with pericardial constriction or restrictive cardiomyopathy [68]. However, Gerson et al. found a considerably greater LV peak filling rate in patients with pericardial constriction [69].

Clements et al. showed that patients with cardiac amyloidosis also had restrictive filling physiology. The aim of their study was to compare LV systolic and diastolic function during inspiration and expiration in four patient groups (patients without cardiac disease, with severe pulmonary disease, with cardiac amyloidosis, and with pericardial constriction). They found some possible clinical diagnostic implications. First, it may be possible with radionuclide ventriculography to use respiratory variations in the LV time to peak filling rate as a marker of pericardial constriction. Second, LV filling measurements may be used to distinguish severe pulmonary disease from cardiac amyloidosis and pericardial constriction [70].

Hongo et al. assessed LV diastolic findings in 17 patients with familial amyloid polyneuropathy and in 20 normal subjects by radionuclide angiography. Peak filling rate was significantly lower and time to peak filling significantly higher in patients than in normal subjects. Most patients had no clinical evidence of heart disease, a normal ventricular wall thickness and a normal ejection fraction, but an abnormality in diastolic filling. Thus, abnormal diastolic filling can be seen even in the early stages of familial amyloid polyneuropathy and may be related to myocardial amyloid deposition [71].

\section{Discussion}

Amyloidosis is a systemic disease that can affect multiple organs and therefore has a poor prognosis. Different types of amyloidosis are related to underlying disease and to deposition in various organs. Several imaging modalities have been proposed for the identification of specific organ involvement and risk stratification. In particular, the added value of identification of cardiac involvement is very high, because of the high rates of arrhythmias, the quick deterioration in cardiac dysfunction and sudden death. Diagnostic imaging of the severity of cardiac amyloidosis is important in the decision making concerning ICD implants or heart transplantation. Therefore, reliable and early detection of cardiac involvement of amyloidosis is important. In this review, we tried to identify all nuclear imaging modalities for the detection of cardiac amyloidosis. Nuclear imaging modalities identify pathophysiological activity which makes it possible to identify organs at risk at an early stage. Another added value of nuclear imaging modalities is the possibility for quantification.

A stepwise approach to diagnosis of and therapy for systemic amyloidosis was presented by Hazenberg et al. [2]. The heart can be examined with electrocardiography (signs of low voltage and pseudoanteroseptal infarction), chest radiography (normal sized heart despite signs of cardiac failure), echocardiography (thickness of septum and ventricular walls), and MUGA scan (LV ejection fraction). But these examinations are all nonspecific for cardiac amyloid involvement.

Two biochemical markers are of importance in cardiac amyloidosis. NT-proBNP (the N-terminal pro-brain natriuretic peptide) appears to be a sensitive marker of myocardial dysfunction and a powerful prognostic determinant in AL amyloidosis. Heart involvement was estimated on the basis of clinical signs, electrocardiography and echocardiography [72]. Another study also concluded that determining levels of circulating biomarkers (NT-proBNP and cardiac troponins) may be a powerful tool for staging 
patients with $\mathrm{AL}$, especially those patients undergoing peripheral blood stem cell transplantation [73].

For specific evaluation systemic amyloidosis, serum amyloid P component (SAP) scintigraphy is a technique that has been developed in London for specific evaluation of amyloidosis [74-77] and is extensively used in our hospital. All amyloid deposits contain SAP, a glycoprotein that binds in a calcium-dependent way to all amyloid deposits independently of the protein of origin. SAP is labelled with ${ }^{123} \mathrm{I}$ and shows specific uptake in the liver, spleen, kidneys, adrenals, bone marrow and joints (for example, see Fig. 2). However, the myocardium does not show specific uptake, probably due to the combination of high background activity of tracer still present in the blood pool and decreased permeability of this tracer in cardiac tissue. Therefore, ${ }^{123} \mathrm{I}-$ SAP scintigraphy is very useful for systemic amyloidosis, but not for identifying cardiac involvement.

With regard to the optimal imaging modality to visualize cardiac amyloid involvement, different imaging methods are available each with a different physiological mechanism. It seems clear that imaging with perfusion tracers ${ }^{99 \mathrm{~m}} \mathrm{Tc}$ sestamibi and ${ }^{201} \mathrm{Tl}$ ) and evaluation of LV function (radionuclide ventriculography) is not the best method, because of their very low specificity. Imaging the metabolism of free fatty acids with ${ }^{123}$ I-BMIPP also has no role in the evaluation of cardiac amyloidosis (limited experience, better results with other radionuclides). Another tracer of metabolism, ${ }^{99 \mathrm{~m}} \mathrm{Tc}(\mathrm{V})$-DMSA has a high physiological uptake in the blood pool and is therefore of very limited use for amyloid depositions in the heart. The best results can be found with radiopharmaceuticals that image amyloid deposits or indirectly by imaging myocardial neuropathy.

Aprotinin labelled with ${ }^{99 \mathrm{~m}} \mathrm{Tc}$ provides an imaging method for amyloid deposits. Because of physiological uptake in the liver, spleen and kidneys it is only useful in extraabdominal disease, such as cardiac depositions. A limited number of studies have been performed with this tracer, but all conclude that ${ }^{99 \mathrm{~m}} \mathrm{Tc}$-aprotinin can be helpful in the diagnosis of amyloid heart disease. Different phosphate derivatives (PYP, MDP, DPD) labelled with ${ }^{99 \mathrm{~m}} \mathrm{Tc}$ seem valuable for detecting cardiac involvement of amyloidosis, but a certain amount of amyloid is necessary. There seems to be no role for this approach in the early detection of cardiac amyloidosis, but it may play a role in the differential diagnosis of ATTR versus AL aetiology in patients with documented cardiac amyloidosis [58]. There is only a limited role for ${ }^{67} \mathrm{Ga}$ for detection amyloid depositions in the heart with no advantages compared to other tracers. One study has shown promising results for ${ }^{111}$ In antimyosin antibody imaging [65]. More studies have to be performed to determine the exact role of this antimyosin antibody in imaging. To date, the best option for imaging amyloid deposits seems to be with ${ }^{99 m} \mathrm{Tc}$ - aprotinin. But all the tracers mentioned previously do not have a very high sensitivity.

The best results we found in the literature are related to the imaging of myocardial innervation by ${ }^{123} \mathrm{I}-\mathrm{MIBG}$.
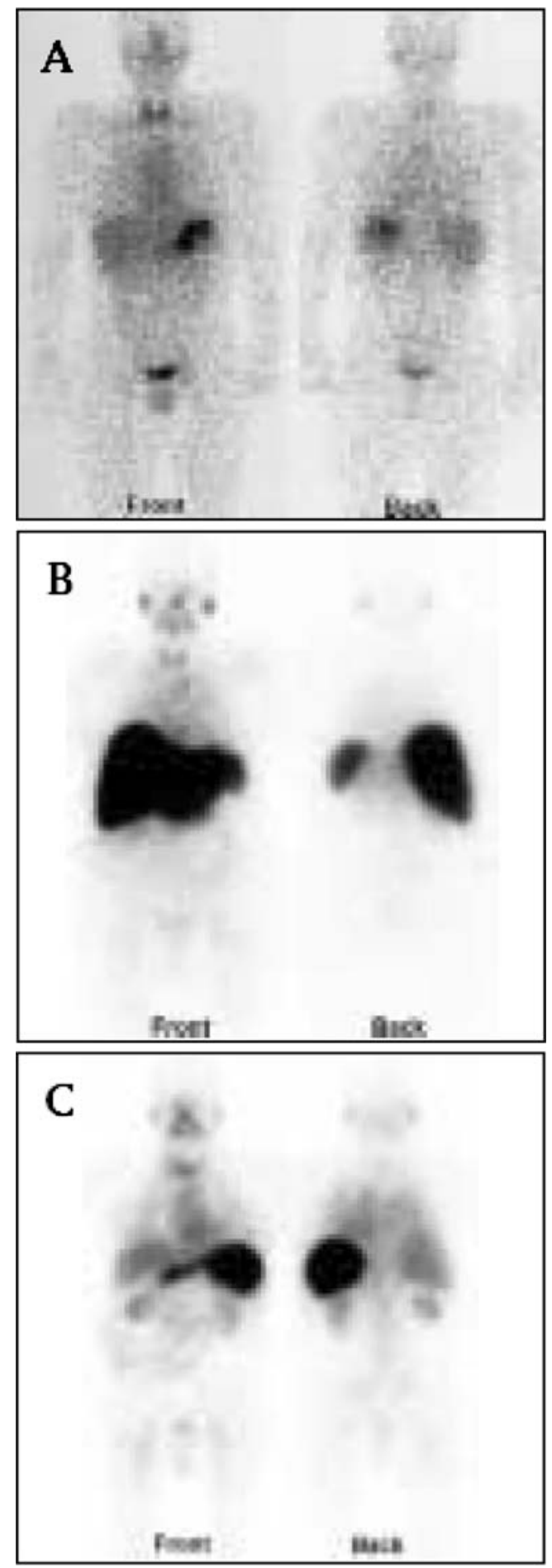

Fig. 2 Serum amyloid P component scintigraphy: a normal patient; b intense uptake in the liver and spleen in a patient with $\mathrm{AL}$ amyloidosis; $\mathbf{c}$ uptake in the spleen and kidney in a patient with AA amyloidosis (Hazenberg et al.) 
MIBG is retained in sympathetic nerve endings, and the localization of MIBG relates to the presence of sympathetic nerves. Myocardial defects in MIBG activity seem to correlate with impaired cardiac sympathetic nerve endings due to amyloid deposits. This can be identified very early in cardiac amyloidosis and shows better results than with other tracers. The clinical severity of disease correlates with a decrease in MIBG uptake. Overall, ${ }^{123}$ I-MIBG is a good option for objective evaluation of cardiac sympathetic level and seems to have an important role in the measurement of amyloid myocardial infiltration.

None of the tracers have been used in the follow-up of disease. We could not find clinical studies concerning the correlation of tracer with the prognosis of disease, but it seems that the amount of decrease in MIBG activity correlates with the severity of amyloid involvement and therefore a worse prognosis. High uptake of Tc-labelled phosphates in the heart correlates with high amounts of amyloid fibrils in the heart and therefore with an advanced stage.

All of the above-mentioned tracers are SPECT tracers. In general, PET tracers will provide absolute quantification and definitely better resolution than SPECT tracers and hence there may be an advantage in using PET tracers for the evaluation of amyloidosis. There are a few PET tracers now available, but none of them is very specific for amyloidosis. ${ }^{18}$ F-FDG, the most widely used PET tracer, is not suitable for evaluating systemic amyloidosis. There are some case reports of positive FDG-PET findings in pulmonary amyloidosis, but with very low specificity [78]. Because of the physiological uptake of FDG in the heart, it is not used in cardiac amyloidosis. ${ }^{11} \mathrm{C}$ - $m$-hydroxyephedrine is useful for sympathetic innervation quantification of the heart (usually in diabetic patients), but is not used for imaging cardiac amyloidosis. ${ }^{11} \mathrm{C}$-Pittsburgh compound-B $(\mathrm{PiB})$ is used with very good results for imaging brain amyloid in Alzheimer's disease by binding to amyloid- $\beta$ deposits $[79,80]$, but this $\beta$ type is specific for the brain and not deposited elsewhere in the body. It is not the tracer of choice for imaging cardiac amyloidosis.

In conclusion, the most validated and most accurate but indirect imaging method in nuclear medicine for the evaluation of amyloid myocardial infiltration is ${ }^{123} \mathrm{I}$-MIBG. PET tracers, despite the big advantages of absolute quantification and higher resolution, are not used for the evaluation of cardiac amyloidosis. This may be an additional clinical challenge in this era of PET proliferation.

\section{Conflict of interest None.}

Open Access This article is distributed under the terms of the Creative Commons Attribution Noncommercial License which permits any noncommercial use, distribution, and reproduction in any medium, provided the original author(s) and source are credited.

\section{References}

1. Selvanayagam JB, Hawkins PN, Paul B, et al. Evaluation and management of the cardiac amyloidosis. J Am Coll Cardiol 2007;50(22):2101-10.

2. Hazenberg BP, van Gameren II, Bijzet J, et al. Diagnostic and therapeutic approach of systemic amyloidosis. Neth J Med 2004;62(4):121-8.

3. Westermark P, Benson MD, Buxbaum JN, et al. Amyloid: toward terminology clarification. Report from the Nomenclature Committee of the International Society of Amyloidosis. Amyloid 2005;12(1):1-4.

4. Dubrey SW, Cha K, Anderson J, et al. The clinical features of immunoglobulin light-chain (AL) amyloidosis with heart involvement. QJM 1998;91:141-57.

5. Dubrey SW, Cha K, Simms RW, et al. Electrocardiography and Doppler echocardiography in secondary (AA) amyloidosis. Am J Cardiol 1996;77:313-5.

6. Benson MD. The hereditary amyloidosis. Best Pract Res Clin Rheumatol 2003;17:909-27.

7. Ng B, Connors LH, Davidoff R, et al. Senile systemic amyloidosis presenting with heart failure: a comparison with light chainassociated amyloidosis. Arch Intern Med 2005;165:1425-9.

8. Camacho V, Carrio I. Targeting neuronal dysfunction and receptor imaging. Curr Opin Biotechnol 2007;18(1):60-4.

9. Hongo M, Urushibata K, Kai R, et al. Iodine-123 metaiodobenzylguanidine scintigraphic analysis of myocardial sympathetic innervation in patients with AL (primary) amyloidosis. Am Heart J 2002;144(1):122-9.

10. Yamashina S, Yamazaki J. Neuronal imaging using SPECT. Eur J Nucl Med Mol Imaging 2007;34(Suppl 1):S62-73.

11. Nakata T, Shimamoto K, Yonekura S, et al. Cardiac sympathetic denervation in transthyretin-related familial amyloidotic polyneuropathy: detection with iodine-12-MIBG. J Nucl Med 1995;36 (6): $1040-2$.

12. Arbab AS, Koizumi K, Toyama K, et al. Scan findings of various myocardial SPECT agents in a case of amyloid polyneuropathy with suspected myocardial involvement. Ann Nucl Med 1997;11 (2):139-41.

13. Tanaka M, Hongo M, Kinoshita O, et al. Iodine-123 metaiodobenzylguanidine scintigraphic assessment of myocardial sympathetic innervation in patients with familial amyloid polyneuropathy. J Am Coll Cardiol 1997;29(1):168-74.

14. Delahaye N, Dinanian S, Slama MS, et al. Cardiac sympathetic denervation in familial amyloid polyneuropathy assessed by iodine-123 metaiodobenzylguanidine scintigraphy and heart rate variability. Eur J Nucl Med 1999;26(4):416-24.

15. Lekakis J, Dimopoulos MA, Prassopoulos V, et al. Myocardial adrenergic denervation in patients with primary (AL) amyloidosis. Amyloid 2003;10(2):117-20.

16. Delahaye N, Rouzet F, Sarda L, et al. Impact of liver transplantation on cardiac autonomic denervation in familial amyloid polyneuropathy. Medicine (Baltimore) 2006;85(4):229-38.

17. Delahaye N, Le Guludec D, Dinanian S, et al. Myocardial muscarinic receptor upregulation and normal response to isoproterenol in denervated hearts by familial amyloid polyneuropathy. Circulation 2001;104:2911-6.

18. Yazaki Y, Isobe M, Takahashi W, et al. Assessment of myocardial fatty acid metabolic abnormalities in patients with idiopathic dilated cardiomyopathy using 123 I BMIPP SPECT: correlation with clinicopathological findings and clinical course. Heart 1999;81(2):153-9.

19. De Geeter F, Franken PR, Knapp FF, et al. Relationship between blood flow and fatty acid metabolism in subacute myocardial infarction: a study by means of $99 \mathrm{mTc}-$ Sestamibi and I-beta- 
methyl-iodo-phenyl pentadecanoic acid. Eur J Nucl Med 1994;21:283-91.

20. Franken PR, De Geeter F, Dendale P, et al. Abnormal free fatty acid uptake in subacute myocardial infarction after coronary thrombolysis: correlation with wall motion and inotropic reserve. J Nucl Med 1994;35:1758-65.

21. Nakata T, Tanaka S, Hamagami S, et al. Detection of impaired fatty acid metabolism and dyskinesis in hypertrophic cardiomyopathy with iodine-123-BMIPP. J Nucl Med 1996;37:167981.

22. Hambye AS, Dobbeleir AA, Vervaet AM, et al. BMIPP imaging to improve the value of sestamibi scintigraphy for predicting functional outcome in severe chronic ischemic left ventricular dysfunction. J Nucl Med 1999;40(9):1468-76.

23. Athanasoulis T, Koutsikos J, Moulopoulos LA, et al. Reverse of the differential uptake intensity of Tc-99m MIBI and Tc-99m VDMSA by multiple myeloma lesions in response to therapy. Clin Nucl Med 2003;28(8):631-5.

24. Papantoniou VJ, Souvatzoglou MA, Valotassiou VJ, et al. Relationship of cell proliferation (Ki-67) to ${ }^{99 \mathrm{~m}} \mathrm{Tc}-(\mathrm{V}) \mathrm{DMSA}$ uptake in breast cancer. Br Cancer Res 2003;6(2):56-62.

25. Kobayashi H, Sakahara H, Itoh $T$, et al. Technetium-99m(V) dimercaptosuccinic acid uptake in intra-abdominal massive deposit of amyloid protein. J Nucl Med 1993;34(5):815-7.

26. Ohta H, Endo K, Kanoh T, et al. Technetium-99m (V) DMSA uptake in amyloidosis. J Nucl Med 1989;30(12):2049-52.

27. Yen TC, Tzen KY, Chen KS, et al. The value of gallium-67 and thallium-201 whole-body and single-photon emission tomography images in dialysis-related $\beta 2$-microglobulin amyloid. Eur J Nucl Med 2000;27(1):56-61.

28. Low YH, Ang ES, Goh AS, et al. Technetium-99m (Tc-99m) diphosphono-propanedicarboxylic acid bone tracer uptake and Tc99m sestamibi distribution in cardiac amyloidosis - a case report. Ann Acad Med Singapore 1995;24(6):898-901.

29. Wechalekar K, Ng FS, Poole-Wilson PA, et al. Cardiac amyloidosis diagnosed incidentally by bone scintigraphy. J Nucl Cardiol 2007;14(5):750-3.

30. Kobayashi T, Sekiya M, Fujiwara Y, et al. A patient with cardiac amyloidosis presenting a rapid increase in technetium-99mhydroxymethylene diphosphonate accumulation. Ann Nucl Med 1993;7(4):273-6.

31. Kodama K, Hamada M, Kuwahara T, et al. Rest-redistribution thallium-201 myocardial scintigraphic study in cardiac amyloidosis. Int J Card Imaging 1999;15(5):371-8.

32. Sojan SM, Smyth DR, Tsopelas C, et al. Pharmacokinetics and normal scintigraphic appearance of $99 \mathrm{mTc}$ aprotinin. Nucl Med Commun 2005;26(6):535-9.

33. Aprile C, Marinone G, Saponaro R, et al. Cardiac and pleuropulmonary AL amyloid imaging with technetium-99m labelled aprotinin. Eur J Nucl Med 1995;22(12):1393-401.

34. Schaadt BK, Hendel HW, Gimsing P, et al. 99mTc-aprotinin scintigraphy in amyloidosis. J Nucl Med 2003;44(2):177-83.

35. Han S, Chong V, Murray $\mathrm{T}$, et al. Preliminary experience of $99 \mathrm{mTc}$-aprotinin scintigraphy in amyloidosis. Eur J Haematol 2007;79(6):494-500.

36. Kula RW, Engel WK, Line BR. Scanning for soft-tissue amyloid. Lancet 1977;1(8002):92-3.

37. Puille M, Altland K, Linke RP, et al. 99mTc-DPD scintigraphy in transthyretin-related familial amyloidotic polyneuropathy. Eur J Nucl Med Mol Imaging 2002;29(3):376-9.

38. Karp K, Naslund U, Backman C, et al. Technetium-99m pyrophosphate single-photon emission computed tomography of the heart in familial amyloid polyneuropathy. Int $\mathrm{J}$ Cardiol 1987;14(3):365-9.

39. Leinonen H, Totterman KJ, Korppi-Tommola T, et al. Negative myocardial technetium- $99 \mathrm{~m}$ pyrophosphate scintigraphy in amy- loid heart disease associated with type AA systemic amyloidosis. Am J Cardiol 1984;53(2):380-1.

40. Kulhanek J, Movahed A. Uptake of technetium 99m HDP in cardiac amyloidosis. Int J Cardiovasc Imaging 2003;19(3):225-7.

41. Casset-Senon D, Secchi V, Arbeille P, et al. Localization of myocardial amyloid deposits in cardiac amyloidosis by Tc-99m pyrophosphate myocardial SPECT: implication for medical treatment. Clin Nucl Med 2005;30(7):496-7.

42. Falk RH, Lee VW, Rubinow A, et al. Cardiac technetium-99m pyrophosphate scintigraphy in familial amyloidosis. Am J Cardiol 1984;54(8):1150-1.

43. Ruder MA, Alpert MA, Sanfelippo JF, et al. Symptomatic cardiac amyloidosis in an American family. South Med J 1984;77(7):831-3.

44. Sobol SM, Brown JM, Bunker SR, et al. Noninvasive diagnosis of cardiac amyloidosis by technetium-99m-pyrophosphate myocardial scintigraphy. Am Heart J 1982;103(4 Pt 1):563-6.

45. Schiff $S$, Bateman T, Moffatt R, et al. Diagnostic considerations in cardiomyopathy: unique scintigraphic pattern of diffuse biventricular technetium-99m-pyrophosphate uptake in amyloid heart disease. Am Heart J 1982;103(4 Pt 1):562-3.

46. Ali A, Turner DA, Rosenbush SW, et al. Bone scintigram in cardiac amyloidosis: a case report. Clin Nucl Med 1981;6(3):1058.

47. Ak I, Vardareli E, Erdinc O, et al. Myocardial Tc-99m MDP uptake on a bone scan in senile systemic amyloidosis with cardiac involvement. Clin Nucl Med 2000;25(10):826-7.

48. Fournier C, Grimon G, Rinaldi JP, et al. Usefulness of technetium$99 \mathrm{~m}$ pyrophosphate myocardial scintigraphy in amyloid polyneuropathy and correlation with echocardiography. Am J Cardiol 1993;72(11):854-7.

49. Hongo M, Hirayama J, Fujii $T$, et al. Early identification of amyloid heart disease by technetium-99m-pyrophosphate scintigraphy: a study with familial amyloid polyneuropathy. Am Heart J 1987;113(3):654-62.

50. Davidson RM, Lin DS, Klima M. Temporal changes in myocardial uptake on serial bone scans in two cases of presumed senile cardiac amyloidosis. Clin Nucl Med 1993;18(10):858-62.

51. Goldstein SA, Lindsay J, Chandeysson PL, et al. Usefulness of technetium pyrophosphate scintigraphy in demonstrating cardiac amyloidosis in persons aged 85 years and older. Am J Cardiol 1989;63(11):752-3.

52. Wizenberg TA, Muz J, Sohn YH, et al. Value of positive myocardial technetium- $99 \mathrm{~m}$-pyrophosphate scintigraphy in the non-invasive diagnosis of cardiac amyloidosis. Am Heart J 1982;103(4 Pt 1):468-73.

53. Falk RH, Lee VW, Rubinow A. Sensitivity of technetium-99mpyrophosphate scintigraphy in diagnosing cardiac amyloidosis. Am J Cardiol 1983;51(5):826-30.

54. Lee VW, Caldarone AG, Falk RH, et al. Amyloidosis of heart and liver: comparison of Tc-99m pyrophosphate and Tc-99m methylene diphosphonate for detection. Radiology 1983;148(1):239-42.

55. Eriksson P, Backman C, Bjerle P, et al. Non-invasive assessment of the presence and severity of cardiac amyloidosis. A study in familial amyloidosis with polyneuropathy by cross sectional echocardiography and technetium-99m pyrophosphate scintigraphy. Br Heart J 1984;52(3):321-6.

56. Janssen S, Piers DA, van Rijswijk MH, et al. Soft-tissue uptake of 99mTc-diphosphonate and 99mTc-pyrophosphate in amyloidosis. Eur J Nucl Med 1990;16(8-10):663-70.

57. Hartmann A, Frenkel J, Hopf R, et al. Is technetium-99mpyrophosphate scintigraphy valuable in the diagnosis of cardiac amyloidosis? Int J Card Imaging 1990;5(4):227-31.

58. Perugini E, Guidalotti PL, Salvi F, et al. Noninvasive etiologic diagnosis of cardiac amyloidosis using 99mTc-3,3-diphosphono1,2-propanodicarboxylic acid scintigraphy. J Am Coll Cardiol 2005;46(6):1076-84. 
59. Bernstein LR. Mechanisms of therapeutic activity for gallium. Pharm Rev 1998;50(4):665-82.

60. Braun SD, Lisbona R, Novales-Diaz JA, et al. Myocardial uptake of 99mTc-phosphate tracer in amyloidosis. Clin Nucl Med 1979;4 (6):244-5.

61. Montes J, Lopez L, Chamorro JL, et al. Cardiac gallium uptake in amyloidosis. Eur J Nucl Med 1984;9(9):438.

62. Li CK, Rabinovitch MA, Juni JE, et al. Scintigraphic characterization of amyloid cardiomyopathy. Clin Nucl Med 1985;10(3):156-9.

63. Jain D, Crawly JC, Lahiri A, et al. Indium-111 antimyosin images compared with triphenyltetrazolium chloride staining in a patient six days after myocardial infarction. J Nucl Med 1990; 31:231-3.

64. Carrio I, Berna L, Ballester M, et al. Indium-111 antimyosin scintigraphy to assess myocardial damage in patients with suspected myocarditis and cardiac rejection. J Nucl Med 1988;29:1893-900.

65. Lekakis J, Vassipoulos N, Psichoyiou H, et al. Doxorubicin cardiotoxicity detected by indium-111 myosin specific imaging. Eur J Nucl Med 1991;18:225-6.

66. Lekakis J, Nanas J, Moustafellou C, et al. Cardiac amyloidosis detected by indium-111 antimyosin imaging. Am Heart J 1992;124:1630-1.

67. Lekakis J, Dimopoulos M, Nanas J, et al. Antimyosin scintigraphy for detection of cardiac amyloidosis. Am J Cardiol 1997;80: 963-5.

68. Aroney CN, Ruddy TD, Dighero H, et al. Differentiation of restrictive cardiomyopathy from pericardial constriction: assessment of diastolic function by radionuclide angiography. J Am Coll Cardiol 1989;13:1007-14.

69. Gerson MC, Colthar MS, Fowler NO. Differentiation of constrictive pericarditis and restrictive cardiomyopathy by radionuclide ventriculography. Am Heart J 1989;118:114-20.

70. Clements IP, Olson LJ, Scanlon PD, et al. The effect of respiration on left ventricular diastolic filling as assessed by radionuclide ventriculography. Nucl Med Commun 2000;21:55-63.
71. Hongo M, Fujii T, Hirayama J, et al. Radionuclide angiographic assessment of left ventricular diastolic filling in amyloid heart disease: a study of patients with familial amyloid polyneuropathy. J Am Coll Cardiol 1989;13:48-53.

72. Palladini G, Campana C, Klersy C, et al. Serum N-terminal probrain natriuretic peptide is a sensitive marker of myocardial dysfunction in AL amyloidosis. Circulation 2003;107:2440-5.

73. Dispenzieri A, Gertz MA, Kyle RA, et al. Prognostication of survival using cardiac troponins and $\mathrm{N}$-terminal pro-brain natriuretic peptide in patients with primary systemic amyloidosis undergoing peripheral blood stem cell transplantation. Blood 2004;104(6):1881-7.

74. Hawkins PN, Lavender JP, Pepys MB. Evaluation of systemic amyloidosis by scintigraphy with 123I-labeled serum amyloid $\mathrm{P}$ component. N Engl J Med 1990;323:508-13.

75. Hazenberg BP, van Rijswijk MH, Piers DA, et al. Diagnostic performance of 123I-labeled serum amyloid P component scintigraphy in patients with amyloidosis. Am J Med 2006;119:355. e15-e24.

76. Jager PL, Hazenberg BP, Franssen EJF, et al. Kinetic studies with iodine-123-labeled serum amyloid $\mathrm{P}$ component in patients with systemic AA and AL amyloidosis and assessment of clinical value. J Nucl Med 1998;39:699-706.

77. Hazenberg BP, Van Rijswijk MH, Lub-de Hooge M, et al. Diagnostic performance and prognostic value of extravascular retention of 123I-labeled serum amyloid $\mathrm{P}$ component in systemic amyloidosis. J Nucl Med 2007;48(6):865-72.

78. Yadav S, Sharma S, Gilfillan I. Unusual positron emission tomography findings in pulmonary amyloidosis: a case report. $\mathrm{J}$ Cardiothorac Surg 2006;1:32.

79. Klunk WE, Engler H, Nordberg A, et al. Imaging brain amyloid in Alzheimer's disease with Pittsburgh Compound-B. Ann Neurol 2004;55(3):306-19.

80. Rabinovici GD, Furst AJ, O'Neil JP, et al. 11C-PIB PET imaging in Alzheimer disease and frontotemporal lobar degeneration. Neurology 2007;68:1205-12. 